\title{
Predictive values of TNF- $\alpha$, IL-6, IL-10 for radiation pneumonitis
}

\author{
B. Li ${ }^{1 \#, ~ S . H . ~ C h e n ~}{ }^{2 \#}$, H.J. Lu3 ${ }^{*}$, Y. Tan3 \\ ${ }^{1}$ Department of Oncology, Jiaozhou Central Hospital, Jiaozhou, Qingdao, Shandong, China \\ 2Department of Oncology, Qingdao Economic Technological Development Area First People's Hospital, Shandong, \\ China \\ ${ }^{3}$ Department of Oncology, the Affiliated Hospital of Qingdao University, Qingdao, Shandong, China
}

\section{- Original article}

*Corresponding author:
Dr. Hai Jun. Lu,
Fax: +86 532 82911348
E-mail: Ihj82920608@163.com
Revised: Oct. 2015
Accepted: Nov. 2015
Int. J. Radiat. Res., July 2016;
14(3): 173-179
DOI: 10.18869/acadpub.ijrr.14.3.173

\# These authors contributed to the work equally and should be regarded as co-first authors.

\begin{abstract}
Background: To investigate the expression of TNF- $\alpha$, IL-6, IL-10 in the plasma of patients with lung cancer who received radiation therapy (RT), and to analyze the correlation between these cytokines and radiation pneumonitis (RP). Materials and Methods: Patients with lung cancer who received 3DCRT in our hospital were prospectively evaluated. Circulating cytokine levels were measured with ELISA before RT and at the end of RT. Regular follow-up was undertaken 3 months after RT. Statistical analysis was applied to determine the relevance of cytokines to radiation-induced lung injury. Results: Of 104 patients, 29 (27.9\%) developed RP. The levels of TNF- $\alpha$ and IL -6 levels in the plasma after RT were significantly higher than before RT $(p<0.05)$, whereas IL -10 levels were significantly lower after RT than before RT $(p<0.05)$. Before RT, TNF- $\alpha$ levels were higher in RP group $(p<0.05)$, but there were no differences in TNF- $\alpha$ levels after RT. No association was observed between IL- 6 and IL-10 levels and the risk of RP. Univariate analysis showed that baseline pulmonary function, smoking history, histopathology, lung volume receiving $\geq 20$ Gy (V20), Mean lung dose (MLD) and total radiation dose were related to $R P$, but only MLD was an independent risk factor for RP in lung cancer patients $(O R>1)$. Conclusion: TNF- $\alpha$ levels in plasma were closely related to RP but still cannot be used as predictors for RP.
\end{abstract}

Keywords: Radiation pneumonitis, tumor necrosis factor-a, interleukin-6, interleukin-10, lung cancer.

\section{INTRODUCTION}

Radiation therapy (RT) plays an important role in the treatment of lung cancer. Increased radiation doses improve local control, but also cause more pulmonary toxicity (1).Radiation pneumonitis (RP) is one of the most serious complications in thoracic radiotherapy, occurring during RT or in the previous 3 months following RT (2). RP limits the patient's ability to finish RT at the prescribed dose and achieve the proper course of treatment. In addition, RP decreases pulmonary function and affects the patient's quality of life. RP is essentially a type of aseptic inflammation, so the condition cannot be reversed. Therefore, preventing RP is vital.

To date, the mechanisms of RP are still obscure. Among the hypotheses, the theory of cytokines attracts the most attention. Studies indicate that the large number of free radicals produced by radiation triggers a series of cascade reactions in the early stage of irradiation $(3,4)$. These free radicals cause dysfunction of vascular endothelial cells, hypoxia, and an increase in vascular permeability and edema $(3,4)$. These changes promote migration, accumulation and activation of monocytemacrophages, which cause oxidative stress and release a mass of cytokines $(3,4)$. And eventually cause apoptosis, collagen deposition and fibrosis $(3,4)$. Cytokines play an important role in this procedure. Basic and clinical research indicate that the cytokines involved in RP can be divided into two categories. TGF- $\alpha$, PDGF, ET-1, EGFR 
and CRP which belong to the first category, promote the proliferation and differentiation of fibroblasts and regulate the degradation of extracellular matrix (5). The second category of cytokines including TNF- $\alpha$, IL-1, IL-6 and IL-10, primarily mediate inflammatory response $(5)$.

TNF- $\alpha$ is both a pro-inflammatory cytokine and a regulator of the cytokine network. Binding of TNF- $\alpha$ to its receptor stimulates multiple signal transduction pathways, inducing expression of a variety of transcription factors and cytokines, growth factors, intercellular adhesion molecule and acute phase proteins $(6,7)$. In addition, TNF- $\alpha$ promotes the growth of fibroblasts and stimulates the synthesis and release of cytokines such as IL-6, IL-1 and IFN, and then produces a cascade effect of cytokines (6,7). TNF- $\alpha$ levels in the lung increase significantly after radiation and radiationinduced pulmonary injury was reduced by lowering the TNF- $\alpha$ level (8). Therefore, TNF- $\alpha$ plays a key role in RP.

IL-6 is synthesized and secreted by various cells in the lung parenchyma, including alveolar macrophages, II -alveolar cells, $\mathrm{T}$ lymphocytes and pulmonary fibroblasts $(9,10)$. IL-6 is an important pleiotropic cytokine that regulates immune responses and inflammatory response by inducing hepatocytes and lung fibroblasts to release CRP, SSA and other acute phase proteins $(9,10)$. IL-6 levels in the serum can be used to judge the inflammatory state of the lung. Nicole et al. showed that the level of IL - 6 is higher in the irradiated side of the lungs than in the nonirradiated side (11). Minako et al. demonstrated that IL-6 KO mice have an inherent resistance to the acute phase of alveolar damage after thoracic irradiation (12).

IL-10 is an anti-inflammatory and antioxidative interleukin related to RP by suppressing TNF- $\alpha$. Research confirmed that the flat wound exhibited by IL-10 knockout fetal mice was an inflammatory reaction involving abnormal collagen deposition, whereas normal skin grafts presented minor inflammation and healed well. Therefore, IL-10 is a negative regulator of fibroblast proliferation and collagen deposition.

Cytokines not only play an important role in the pathogenesis and pathological process of RP, but are also meaningful in predicting RP. In this study, we discuss the predictive effect of inflammatory cytokines. Research has demonstrated that drugs such as ulinastatin can decrease the circulating levels of these cytokines and reduce the incidence and grade of RP (13). To some degree, this demonstrates a correlation between cytokines and RP. Research has shown that in the process of acute lung injury, IL-6, TNF $-\alpha$ and other inflammatory cytokines can predict the severity of lung injury accurately (14). In addition, there is a relationship between the expression levels of IL-6, IL-10 and other inflammatory cytokines and occurrence of radiation-induced lung injury in thoracic RT (15). However, it is still controversial whether these cytokines can be used to predict RP. Therefore, we investigated the correlation between cytokines (TNF- $\alpha$, IL-6, IL-10) levels and the occurrence of RP, and evaluated risk factors for RP in lung cancer patients. Thus, individuals at high-risk for RP can be identified before RT, thereby reducing the incidence of RP in patients receiving RT.

\section{MATERIALS AND METHODS}

\section{Clinical data and ethics statement}

Patients with lung cancer who received thoracic conformal radiotherapy in Jiaozhou Central Hospital from January 2013 to September 2014 were prospectively evaluated. Exclusion criteria included under the age of 18 years, life expectancy less than 6 months, Karnofsky Performance Status $\leq 60 \%$, history of radiation treatment. All patients signed an informed consent form before registration. The procedures were approved by an independent protocol review committee and ethics committee in Qingdao University.

\section{Radiotherapy}

All the patients received 3D conformal radiotherapy. The radiotherapy planning varied among the patients in accordance with their actual condition. Generally, clinical tumor volume included gross tumor volume and related lymph 
node regions. The dose was 60 to $70 \mathrm{~Gy}$ in 2.0 to 2.5 Gy daily fractions over 6 to 7 weeks for inoperable patients and 45-58 Gy for patients who received surgical intervention. The mean lung dose (average dose to the CT-defined total lung volume, excluding the gross volume) and V20 (lung volume receiving $\geq 20$ Gy) were calculated from lung DVHs (Dose-volume histogram parameters). Mean lung dose (MLD) was restricted to $<20$ Gy and total lung V20 was confined to $\leq 35 \%$.

\section{Cytokine analysis}

The levels of TNF- $\alpha$, IL-6, and IL-10 were measured before RT and at the end of RT. To obtain plasma, $2 \mathrm{ml}$ of venous blood was collected from the fasting patients and centrifuged at $3000 \mathrm{rpm}$ for 10 minutes at low temperature. The plasma samples were divided into three tubes, and stored at $-70^{\circ} \mathrm{C}$. TNF- $\alpha$, IL- 6 and IL-10 levels were measured by ELISA according to the manufacturer's instructions.

\section{Evaluation of radiation pneumonitis}

To evaluate lung injury and the effect of irradiation, physical examinations were performed by at least two radiation oncologists weekly during the course of RT and once every month for 3 months after RT. A chest computed tomography scan was performed before RT and at each monthly follow-up evaluation after RT. The diagnosis of RP was concluded from clinical symptoms (dry cough, low-grade fever, chest pain and shortness of breath), imaging tests (CT imaging include a diffuse haziness or fuzziness in the areas of the irradiated lung, which form a relatively sharp edge corresponding to the shape and size of the radiation field. The haziness does not disappear after one week's treatment of antibiotic and pulmonary function, in accordance with RTOG (16) for acute RP. Pneumonitis grading was according to the National Cancer Institute Common Toxicity Criteria 4 (17). In our study, a grade $\geq 1$ was considered as RP.

\section{Statistical analysis}

Data were analyzed using SPSS software (version 19.0). Student's t-test was used to compare two group's mean values, and the chisquare test was used for qualitative data, if not appropriate, non-parametric should be used. Logistic regression analysis was performed for seeking predictive factors of RP. P values $<0.05$ were considered to be statistically significant, and all tests were two-sided.

\section{RESULTS}

\section{Follow-up result}

There were 107 patients enrolled in this study. Among these patients, two patients died due to cancer progression and pulmonary inflammation, and one patient was lost during follow-up. Nine of the remaining 104 patients developed RP during RP, and 20 patients developed RP after RT. The incidence of RP was therefore $27.9 \%$. According to the RTOG grading standard, 10 patients had grade 3 RP, 11 patients had grade $2 \mathrm{RP}$, and 8 patients had grade $1 \mathrm{RP}$. The frequencies of grades 3,2 and 1 RP were 9.6\%, $10.6 \%$ and $7.7 \%$, respectively. No cases of grade 4 RP were recorded.

\section{Patient characteristics and radiation pneumonitis}

Multiple characteristics of the patients are listed in table 1 . The age of the patients ranged from 26 to 80 years, with a median age was 59.52 and a mean age of $62.19+11.64$. There were 63 patients $(60.58 \%)$ older than 65 years. Fifty-four patients $(51.92 \%)$ had small cell lung cancer. Surgery was performed in 32 patients $(30.77 \%)$. Fifty-six patient's cases $(53.85 \%)$ received concurrent chemotherapy based on cisplatin, with or without paclitaxel, etoposide or pemetrexed. Thirty-five patients (33.65\%) received chemotherapy before RT, but no more than two treatments. Only 13 patients (12.50\%) received radiotherapy alone. Forty-one patients $(39.42 \%)$ received a total dose $<60 \mathrm{~Gy}$, and 63 patients $(60.58 \%)$ received a total dose $>60 \mathrm{~Gy}$. The median MLD was 15.8Gy, and the median V20 was $27.8 \%$. Univariate analysis indicated that baseline pulmonary function, smoking history, histopathology, V20, MLD, and total

Int. J. Radiat. Res., Vol. 14 No. 3, July 2016 


\section{Li et al. / Predictive values of cytokines for radiation pneumonitis}

radiation dose correlated with RP. The relationship between clinical factors and the occurrence of RP is listed in table 1.

\section{Cytokine levels and radiation pneumonitis}

Table 2 shows the levels of TNF- $\alpha$, IL- 6 and IL-10 in the plasma before and after RT.TNF- $\alpha$ and IL-6 levels were significantly increased after RT, whereas IL-10 was significantly decreased. The levels of IL-10 varied widely. We then analyzed the differences in these three cytokines between the patients who developed RP and the patients that did not. There were no differences in the baseline values between the two groups except for TNF- $\alpha$. Furthermore, the levels of the three cytokines after RT were not significantly different between the two groups. The baseline level of TNF- $\alpha$ was higher in the group that developed RP, and expressed individual differences. The effects of cytokines on RP are shown in tables 3 and 4.

Table 1. Patient characteristics and their relationship with the occurrence of RP.

\begin{tabular}{|c|c|c|c|c|}
\hline \multicolumn{2}{|c|}{ Characteristics } & $R P(n=29)$ & Non-RP $(n=75)$ & $P$ \\
\hline \multicolumn{5}{|c|}{ Age } \\
\hline$<65$ & 41 & 9 & 32 & 0.371 \\
\hline$\geqq 65$ & 63 & 20 & 43 & \\
\hline \multicolumn{5}{|l|}{ Sex } \\
\hline Male & 50 & 17 & 33 & 0.197 \\
\hline Female & 54 & 12 & 42 & \\
\hline \multicolumn{5}{|c|}{ Karnofsky Performance Status } \\
\hline$<80$ & 34 & 11 & 23 & 0.493 \\
\hline$\geq 80$ & 70 & 18 & 52 & \\
\hline \multicolumn{5}{|c|}{ Pulmonary function } \\
\hline Normal & 68 & 8 & 60 & 0.000 \\
\hline Abnormal & 36 & 21 & 15 & \\
\hline \multicolumn{5}{|c|}{ Smoking history } \\
\hline Yes & 74 & 16 & 58 & 0.025 \\
\hline No & 30 & 13 & 17 & \\
\hline \multicolumn{5}{|c|}{ Histopathology } \\
\hline NSCLC & 50 & 19 & 31 & 0.027 \\
\hline SCLC & 54 & 10 & 44 & \\
\hline \multicolumn{5}{|c|}{ Clinical stage } \\
\hline II & 45 & 10 & 35 & 0.398 \\
\hline III & 32 & 9 & 23 & \\
\hline $\mathrm{IV}$ & 27 & 10 & 17 & \\
\hline \multicolumn{5}{|c|}{ Previous surgery } \\
\hline Yes & 32 & 12 & 20 & 0.157 \\
\hline No & 71 & 17 & 54 & \\
\hline \multicolumn{5}{|c|}{ Chemotherapy } \\
\hline Concurrent & 56 & 20 & 36 & 0.150 \\
\hline Sequential & 35 & 7 & 28 & \\
\hline No & 13 & 2 & 11 & \\
\hline \multicolumn{5}{|c|}{ Total dose of RT } \\
\hline$<60 G y$ & 41 & 7 & 34 & 0.047 \\
\hline$\geq 60 \mathrm{~Gy}$ & 63 & 22 & 41 & \\
\hline \multicolumn{5}{|l|}{ V20 } \\
\hline median & 27.8 & 29.3 & 27.2 & 0.008 \\
\hline \multicolumn{5}{|l|}{ MLD } \\
\hline$\overline{\text { median }}$ & 15.8 & 16.8 & 15.4 & 0.000 \\
\hline
\end{tabular}

Int. J. Radiat. Res., Vol. 14 No. 3, July 2016 
Table 2. The levels of TNF- $\alpha$, IL-6 and IL-10 in all patients before and after RT.

\begin{tabular}{|c|c|c|c|c|}
\hline Cytokine & Pre-RT (pg/ml) & Post-RT $(\mathrm{pg} / \mathrm{ml})$ & $\boldsymbol{t}$ & $\boldsymbol{P}$ \\
\hline TNF- $\alpha$ & $30.29 \pm 6.29$ & $47.92 \pm 11.64$ & 7.625 & 0.000 \\
\hline IL-6 & $10.29 \pm 3.17$ & $17.06 \pm 5.92$ & 9.478 & 0.000 \\
\hline IL-10 & $98.06 \pm 17.40$ & $47.14 \pm 13.52$ & 8.146 & 0.000 \\
\hline
\end{tabular}

Table 3. The levels of TNF- $\alpha$, IL- 6 and IL-10 before RT in patients who developed RP and patients who did not develop RP.

\begin{tabular}{|c|c|c|c|c|}
\hline Cytokines & $\mathbf{R P}(\mathbf{n}=\mathbf{2 9})(\mathbf{p g} / \mathbf{m l})$ & Non-RP $(\mathbf{n}=\mathbf{7 5})(\mathbf{p g} / \mathbf{m l})$ & $\boldsymbol{t}$ & $\boldsymbol{P}$ \\
\hline TNF- $\alpha$ & $35.92 \pm 13.20$ & $22.94 \pm 8.12$ & 8.746 & 0.000 \\
\hline IL-6 & $9.82 \pm 4.17$ & $11.04 \pm 4.59$ & 0.286 & 0.752 \\
\hline IL-10 & $46.93 \pm 10.84$ & $48.36 \pm 11.29$ & 0.449 & 0.512 \\
\hline
\end{tabular}

Table 4. The levels of TNF- $\alpha$, IL- 6 and IL-10 after RT in patients who developed RP and patients who did not develop RP.

\begin{tabular}{|c|c|c|c|c|}
\hline Cytokines & $\mathbf{R P}(\mathbf{n}=\mathbf{2 9})(\mathbf{p g} / \mathrm{ml})$ & Non-RP(n=75) $(\mathbf{p g} / \mathrm{ml})$ & $\boldsymbol{t}$ & $\boldsymbol{P}$ \\
\hline TNF- $\alpha$ & $46.82 \pm 12.57$ & $48.49 \pm 11.63$ & 1.024 & 0.916 \\
\hline IL-10 & $95.82 \pm 13.17$ & $104.49 \pm 18.06$ & 0.413 & 0.510 \\
\hline IL-6 & $18.26 \pm 5.40$ & $16.94 \pm 4.36$ & 0.279 & 0.794 \\
\hline
\end{tabular}

\section{Predictive factors of radiation pneumonitis}

Baseline level of TNF- $\alpha$, baseline pulmonary function, smoking history, concurrent chemotherapy, histopathology, V20, MLD and total radiation dose were evaluated in the multivariate analysis. Logistic regression analysis indicated that none of these elements was independent prognostic factors for RP, except MLD. The OR was 1.912 and the 95\% confidence interval was 1.014 to 2.579 .

\section{DISCUSSION}

$\mathrm{RP}$ is the most important dose-limited toxicity. According to reports, the incidence of acute radioactive lung injury is $5 \%$ to $37 \%$. Approximately $10 \%$ to $20 \%$ of non-small-cell lung cancer (NSCLC) patients developed severe lung toxicity after thoracic irradiation, and about $1.6 \%$ of these patients died from RP (18-21). In this study, the incidence of RP was $27.9 \%$ and the incidence of grade $3 \mathrm{RP}$ was about 9.6\%, which consistent with reported results. No RP-related deaths occurred in our study. Although two patients died, there was no clear evidence indicating that the deaths were due to RP.

We have discussed a link between cytokines and RP. Research indicates that mice deficient in TNF- $\alpha$ or the TNF receptor exhibit reduced apoptosis induced by irradiation and pulmonary damage $(22,23)$. Rube et al showed that there are two peaks of IL-6 expression when C57BL/6 mice receive 12 Gy radiation, the first peak appeared within 1-6 $\mathrm{h}$ and the second peak appeared after 8 weeks, coincident with the development of RP (24).In our research, the plasma levels of TNF- $\alpha$ and IL- 6 were greater after RT than before RT. The plasma level of IL10 decreased after RT, indicated that irradiation can cause changes in IL-10.These results are consistent with previous studies and further demonstrate the importance of these cytokines in the development of RP.

Although TNF- $\alpha$, IL-6 and IL-10 play a curious role in RP, whether they should be regarded as predictive factors of RP is controversial. Arpin et al. indicated that early variations in circulating IL-6 and IL-10 levels during 3D-CRT are significantly associated with the risk of RP and may serve as independent predictive factors for this complication (15).

Int. J. Radiat. Res., Vol. 14 No. 3, July 2016 


\section{Li et al. / Predictive values of cytokines for radiation pneumonitis}

RTOG 91-03 indicated that elevations in serum IL-6 levels after 10 Gy of lung irradiation can predict grade $\geqq 2$ acute lung toxicity ${ }^{(25)}$. Chen et al. found that IL-6 levels were significantly higher before, during and after irradiation in patients with RP (26). A meta-analysis revealed that IL-6 levels may correlate with the risk of RP in Caucasians with lung cancer, but the relationship is mild in Asians (27).

In our study, the baseline serum levels of IL6 and IL-10 were similar between the RP group and non-RP groups. Furthermore, the levels of the three cytokines after RT were similar between the two groups. The baseline TNF- $\alpha$ level was higher in the RP group, and expressed individual differences. However, logistic regression analysis indicated that none of these elements were predictors for RP. Our result are consistent with those from Rube et al. who found that plasma TNF- $\alpha$ and IL-6 levels did not correlate with RP during a 9 month follow-up (28). Other studies also found no significance correlation between these three cytokines and RP $(29,30)$. Toshiyuki et al. showed that IL-6 receptor antibody treatment does not ameliorate RP, even with a higher dose and longer course of treatment (31). The primary reason for these differences is that cytokines are produced both in normal lung tissue after irradiation and in NSCLC tumor cell (28). Fu et al noted that ethnicity maybe another reason of these differences, due to the differences in environments, genetic backgrounds and risk factors related to the lifestyles among the populations (27). Furthermore, the differences in experimental methodology and patient eligibility may contribute to this discrepancy. Therefore, more prospective, large sampled and strictly designed trials should be performed.

The results of this study show that there is a close relationship between the total dose of RT and the occurrence of RP. A total dose of $\geqq 60$ Gy may increase the risk of RP. In addition, dose-volume histogram parameters, such as MLD and V20, have been shown to be predictors of RP $(21,29)$, which is consistent with our results. The incidence of RP is higher in nonsmall cell lung cancer than in small cell lung cancer. Perhaps target volume and dose distribution lead to the differences in the incidence of RP and the poor comparability. As for clinical factors, the differences in the type of lung cancer, pulmonary function before treatment and total dose of RT between the two groups (RP and non-RP) were statistically significant. Therefore, combining dose-volume histogram parameters with biological factors, while taking clinical factors into consideration can effectively improve our ability to predict RP.

\section{Conflict of interest: Declared None.}

\section{REFERENCES}

1. Bodnar M, Szylberg L, Kazmierczak W, et al. (2012) Differentiated expression of membrane type metalloproteinases (MMP-14, MMP-15) and pro-MMP2 in laryngeal squamous cell carcinoma. A novel mechanism. J Oral Pathol Med, 23(8): 231-234.

2. Ghafoori P, Marks LB, Vujaskovic Z et a1. (2008) Radiationinduced lung injury, Assessment, management, and prevention. Oncology, 22(1): 37-47.

3. Luzina IG, Todd NW, Sundararajan S, Atamas SP (2015) The cytokines of pulmonary fibrosis: Much learned, much more to learn. Cytokine, 74(1): 88-100.

4. Ding NH, Li JJ, Sun LQ (2013) Molecular mechanisms and treatment of radiation-induced lung fibrosis. Curr Drug Targets, 14(11): 1347-56.

5. Rube CE, Rodemann HP, Rube C (2004) The relevance of cytokines in the radiation-induced lung reaction. Experimental basis and clinical significance. Strahlenther Onkol, 180(9): 541-9.

6. Redlich CA, Gao X, Rockwell S, Kelley M, Elias JA (1996) IL-11 enhances survival and decreases TNF production after radiation induced thoracic injury. J Immunol, 157: 170510.

7. Luo Y, Wang M, Pang Z, Jiang F, Chen J, Zhang J (2013) Locally instilled tumor necrosis factor alpha antisense oligonucleotide contributes to inhibition of TH 2-driven pulmonary fibrosis via induced CD4+ CD25+ Foxp3+ regulatory T cells. J Gene Med, 15(11-12): 441-52.

8. Chiang CS, Liu WC, Jung SM, Chen FH, Wu CR, McBride WH et al. (2005) Compartmental responses after thoracic irradiation of mice: strain differences. Int $J$ Radiat Oncol Biol Phys, 62: 862-71.

9. Wolf J, Rose-John S, Garbers C (2014) Interleukin-6 and its receptors: a highly regulated and dynamic system. Cytokine, 70: 11-20.

10. Scheller J, Chalaris A, Schmidt-Arras D, Rose-John S (2011) The pro- and anti-inflammatory properties of the cytokine interleukin-6. Biochim Biophys Acta, 1813(5): 878-88. 
11. Nicole BB, Lionel BE, Didier C, et al. (2004) Increased IL-6 and TGF- $\beta$ concentrations in bronchoalveolar lavage fluid associated with thoracic radiotherapy. Radiation Oncology Biol Phys, 58: 758-767.

12. Minako S, Mayumi I, Yoichiro I, Toshie O, Hirohiko T, Takashi I (2008) CD44 and bak expression in IL-6 or TNFalpha gene knockout mice after whole Lung irradiation. $J$ Radiat Res, 48: 409-416.

13. Bao P, Zhao W, Li Y et al. (2015) Protective effect of ulinastatin in patients with non-small cell lung cancer after radiation therapy: a randomized, placebo-controlled study. Med Oncol, 32: 405.

14. Chen $Y$, Hyrien $O$, Williams J, Okunieff $P$, Smudzin T, Rubin $P$ (2005) Interleukin (IL)-1A and IL-6:applications to the predictive diagnostic testing of radiation pneumonitis. Int $J$ Radiat Oncol Biol Phys, 62: 260-6.

15. Arpin D, Perol D, Blay JY, Falchero L, Claude L, VuillermozBlas S et al. (2005) Early variations of circulating interleukin-6 and interleukin-10 levels during thoracic radiotherapy are predictive for radiation pneumonitis. $J$ Clin Oncol, 23: 8748-56.

16. Bradley JD, Hope A, El Naqa I, Apte A, Lindsay PE, Bosch WA et al. (2007) Nomogram to predict radiation pneumonitis, derived from a combined analysis of RTOG 9311 and institutional data. Int J Radiat Oncol Biol Phys, 69 (4): 985-92.

17. Gravs PR, Siddiqui F, Anscher MS, Movsas B (2010) Radiation pulmonary toxicity: from mechanisms to management. Semin Radiat Oncol, 20: 201-7.

18. Schallenkamp JM, Miller RC, Brinkmann DH, Foote T, Garces YI (2007) Incidence of radiation pneumonitis after thoracic irradiation: Dose-volume correlates. Int J Radiat Oncol Biol Phys, 67: 410-416.

19. Wang YS, Chang HJ, Chang YC, Huang SC, Ko HL, Chang CC (2013) Serum amyloid a as a predictive marker for radiation pneumonitis in lung cancer patients. Int J Radiat Oncol Biol Phys, 85: 791-7.

20. Yilmaz S, Adas YG, Hicsonmez A, Andrieu MN, Akyurek S, Gokce SC et al. (2014) Evaluation of the radiation pneumonia development risk in lung cancer cases. Asian Pac J Cancer Prev, 15: 7371-7375.

21. Zhuang $\mathrm{H}$, Hou H, Yuan Z, Wang J, Pang Q, Zhao L et al. (2014) Preliminary analysis of the risk factors for radiation pneumonitis in patients with non-small-cell lung cancer treated with concurrent erlotinib and thoracic radiotherapy. Onco Targets Ther, 7: 807-13.

22. Zhang $M$, Qian J, Xing $X$, Kong F-M, Zhao L, Chen $M$ et al. (2008) Inhibition of the tumor necrosis factor-A pathway is radioprotective for the lung. Clin Cancer Res, 14(6): 186876.

23. Hill RP, Zaidi A, Mahmood J, Jelveh S (2011) Investigations into the role of inflammation in normal tissue response to irradiation. Radiother Oncol, 101: 73-79.

24. Rube CE, Uthe D, Wilfert F, Ludwig D, Yang K, Konig J et al. (2005) The bronchiolar epithelium as a prominent source of pro-inflammatory cytokines after lung irradiation. Int $J$ Radiat Oncol Biol Phys, 61: 1482-92.

25. Hartsell WF, Scott CB, Dundas GS, Mohiuddim M, Meredith RF, Rubin P (2007) Can serum markers be used to predict acute and late toxicity in patients with lung cancer? Analysis of RTOG 91-03. Am J Clin Oncol, 30: 368-76.

26. Chen Y, Rubin P, Wiliams J, Hernady E, Smudzin T, Okuniefe P (2001) Circulating IL-6 as a predictor of radiation pneumonitis. Int J Radiation Oncology Biol.Phys, 49: 641648.

27. Fu ZZ, Peng Y, Cao LY, Chen Y-S, Li Kun, Fu B-H (2016) Correlations between serum IL- 6 levels and radiation pneumonitis in lung cancer patients: A Meta-Analysis. J Clin Lab Anal, 30(2): 145-154.

28. Rube CE, Palm J, Erren M, Fleckenstein J. Konig J, Remberger K et al. (2008) Cytokine plasma levels: reliable predictors for radiation pneumonitis? PLOS One $\mathbf{3}$ (8):e2898.

29. Stenmark MH, Cai XW, Shedden K, Hayman JA, Yuan S, Ritter T (2012) Combining physical and biological parameters to predict radiation-induced lung toxicity in patients with non-small-cell lung cancer treated with definitive radiation therapy. Int J Radiat Oncol Biol Phys, 84 (2): e217-22.

30. Kim JY, Kim YS, Kim YK, Park HJ, Kim SJ, Kang JH et al. (2009) The TGF-beta1 dynamics during radiation therapy and its correlation to symptomatic radiation pneumonitis in lung cancer patients. Radiat Oncol, 4: 59.

31. Ogata $T$, Yamazaki $H$, Teshima $T$, Tsuchiya $T$, Nishimoto $N$, Matsuura, N (2012) Anti-IL-6 receptor antibody does not ameliorate radiation pneumonia in mice. Exp Ther Med; 4: 273-276. 
